\title{
Electron imaging of dielectrics under simultaneous electron-ion irradiation
}

\author{
M. Toth ${ }^{\text {a) }}$ \\ Polymers and Colloids Group, Cavendish Laboratory, University of Cambridge, Madingley Road, \\ Cambridge CB3 OHE, United Kingdom \\ M. R. Phillips \\ Microstructural Analysis Unit, University of Technology, Sydney, Broadway, NSW 2007, Australia \\ B. L. Thiel and A. M. Donald \\ Polymers and Colloids Group, Cavendish Laboratory, University of Cambridge, Madingley Road, \\ Cambridge CB3 OHE, United Kingdom
}

(Received 22 October 2001; accepted for publication 4 December 2001)

\begin{abstract}
We demonstrate that if charging caused by electron irradiation of an insulator is controlled by a defocused flux of soft-landing positive ions, secondary electron (SE) images can contain contrast due to lateral variations in (i) changes in the SE yield caused by subsurface trapped charge and (ii) the SE-ion recombination rate. Both contrast mechanisms can provide information on microscopic variations in dielectric properties. We present a model of SE contrast formation that accounts for localized charging and the effects of gas ions on the SE emission process, emitted electrons above the sample surface, and subsurface trapped charge. The model explains the ion flux dependence of charge-induced SE contrast, an increase in the sensitivity to surface contrast observed in SE images of charged dielectrics, and yields procedures for identification of contrast produced by localized sample charging. (C) 2002 American Institute of Physics. [DOI: 10.1063/1.1448875]
\end{abstract}

\section{INTRODUCTION}

Secondary electron (SE) contrast caused by variations in surface potential and in the height of the surface barrier is routinely used to visualize lateral variations in the electronic structure of dielectric-metal composites, semiconductors and semiconductor devices, and superconductors. ${ }^{1-6}$ Voltage and temperature distributions across passivated devices can be imaged due to the effects of the charge/temperature state of device components on localized charging and SE emission from a thin insulating passivation overlayer. ${ }^{7-9}$ However, because of specimen charging artifacts encountered in high vacuum scanning electron microscopes (SEMs), ${ }^{10}$ analogous investigations of bulk insulators have been limited to a few special cases like imaging of defect structures in flatpolished polycrystalline diamond films. ${ }^{11}$ Such features in electron images of dielectrics have been ascribed to a chargeinduced SE contrast mechanism. ${ }^{11}$ However, the usefulness of these imaging modes is usually limited by severe distortions caused by excessive charging. An increasingly popular method of alleviating specimen charging artifacts entails irradiation of the sample by a delocalized flux of soft-landing positive ions, ${ }^{12}$ the approach utilized in variable pressure SEMs. ${ }^{13}$ The magnitude of charging artifacts in electron images can be varied by operating parameters such as gas pressure, SE detector bias, and sample-detector separation. ${ }^{14}$ It is possible to achieve conditions whereby dielectrics exhibit a degree of charging that is sufficiently intense to produce stable SE contrast caused by lateral variations in trapped charge density, but too weak to give rise to chronic charging artifacts that dominate over useful image contrast. Contrast

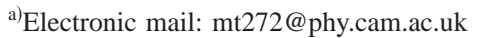

related to localized charging of insulators in a low vacuum environment has been noted by Danilatos ${ }^{13}$ and Holger and Füting ${ }^{15}$ and has subsequently been utilized for visualization of lateral variations in the dielectric properties of $\mathrm{GaN},{ }^{16}$ entirely liquid emulsion systems, ${ }^{17}$ and minerals such as gibbsite and zircon. ${ }^{18,19}$ However, at present, the literature is devoid of detailed studies of the role of the partially ionized gas in the formation of charge-induced SE contrast and microscopic models of charge neutralization in variable pressure SEMs. The need for such models is highlighted by the lack of rigorous explanations of image contrast corresponding to, for example, ferroelectric domains in $\mathrm{LiTaO}_{3},{ }^{20}$ and crystal growth histories in minerals. ${ }^{19}$

In this article we report the results of experiments designed to elucidate SE contrast mechanisms unique to samples irradiated by a flux of positive ions during image acquisition. We present a model that accounts for changes in the surface potential and SE escape barrier caused by the electric field generated by subsurface trapped charge and by ions above the sample surface, and recombination of ions with electrons in the sample and with emitted SEs. We discuss consequent effects on electron emission and detection. The model is used to explain the ion flux dependence of SE contrast produced by localized charging and the presence of unusually high levels of surface contrast in SE images of charged dielectrics.

\section{BACKGROUND THEORY}

\section{A. Low vacuum SEM}

Here we briefly outline aspects of low vacuum SEM and electron-ion recombination theory required for interpretation of data presented in Sec. IV. A schematic illustration of a low 


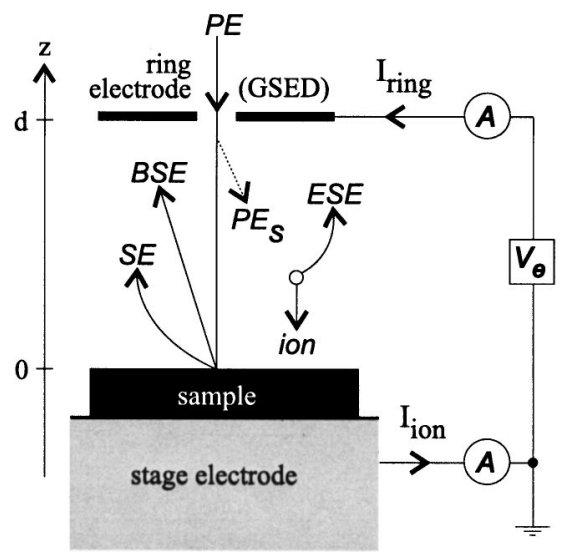

FIG. 1. Schematic illustration of a variable pressure SEM specimen chamber. The ring electrode [the electron collector of the gaseous secondary electron detector, (GSED)] is positively biased with respect to the specimen stage. The directions of motion of charge carriers are shown in the figure (PE: primary electron, $\mathrm{PE}_{s}$ : skirt electron, BSE: backscattered electron, $\mathrm{SE}$ : secondary electron, and ESE: environmental SE). Also shown are the imaging signals, $I_{\text {ring }}$ and $I_{\text {ion }}$, induced in the ring and stage electrodes by the motion of charge carriers.

vacuum SEM chamber is shown in Fig. 1. An electrode, usually a metal ring mounted above the specimen, centered on the optic axis of the microscope, is typically biased by 50-600 V. The high energy primary beam and backscattered electrons (PEs and BSEs) ${ }^{21}$ are sufficiently energetic to ionize gas molecules and are practically unaffected by the detector field. Conversely, the low energy secondary electrons (by definition, $\varepsilon_{\mathrm{SE}}<50 \mathrm{eV}$, but most emitted SEs possess energies of only a few $\mathrm{eV})^{10,22}$ are accelerated by the detector field to energies in excess of the gas ionization threshold. Electrons produced in inelastic electron-gas molecule collisions, so called "environmental" SEs (ESEs), are also accelerated by the field, thus giving rise to a gas ionization cascade that acts as a high gain electron signal amplifier. ${ }^{12,14,23}$ The motion of charge carriers in the chamber induces current flow in the electrode, $I_{\text {ring }}$, (see Fig. 1), often used for electron imaging. ${ }^{14}$ An analogous signal induced in the grounded specimen stage (the so-called "ion current," $I_{\text {ion }}$ ) can also be used for imaging. ${ }^{24,25}$

Gas gain, the mean number of electron-ion pairs produced by each electron injected into the gas, can be approximated by assuming a constant electric field between the sample and the biased electrode. ${ }^{23}$ Gas amplification profiles thus calculated as a function of water vapor pressure for a number of potential differences, $V_{\text {gap }}$, across the gap between the sample and the biased electrode are shown in Fig. $2\left[\mathrm{PE}\right.$ accelerating voltage $\left(\varepsilon_{P E}\right)=15 \mathrm{keV}$, sampleelectrode separation $(d)=2 \mathrm{~mm}$, SE yield $(\delta)=0.2$, and BSE yield $(\eta)=0.04]$. The analytic model used to obtain the profiles, derived and discussed in Ref. 23, does not account for the effects of ions on gas gain discussed in this article. The curves merely serve to illustrate generic trends in the pressure and electric field strength dependencies of the ion generation rate which must be known for correct interpretation of the SE image contrast behavior discussed in Sec. IV.

Ions produced in the cascade drift towards the sample surface where they can recombine with electrons in the

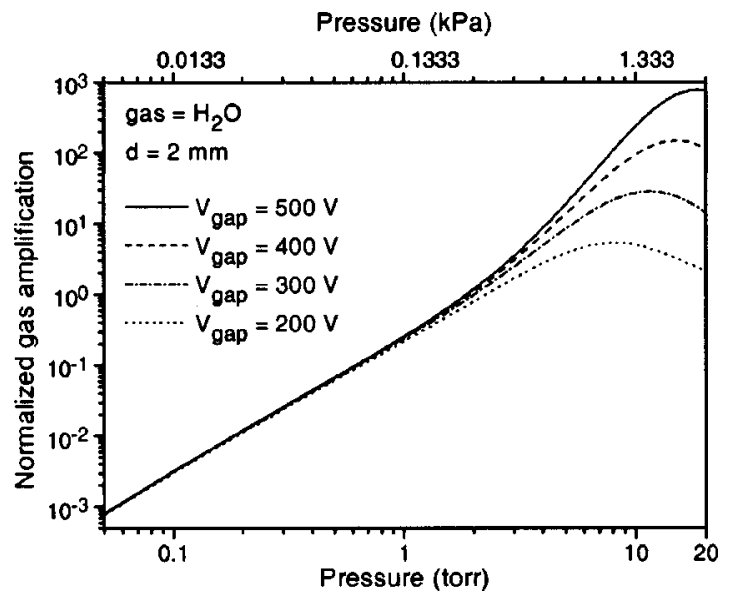

FIG. 2. Gas cascade amplification profiles normalized to the electron beam current. The curves illustrate the general pressure and field strength dependencies of the ion generation rate in a variable pressure SEM chamber [ gas $=\mathrm{H}_{2} \mathrm{O}, d=2 \mathrm{~mm}, V_{\text {gap }}=$ potential drop across the gap between the ring electrode and the sample surface, as shown in Fig. 1, $\varepsilon_{\mathrm{PE}}=15 \mathrm{keV}$, SE yield $(\delta)=0.2$, BSE yield $(\eta)=0.04]$.

specimen, or with emitted SEs. ${ }^{26-31}$ A number of possible electron transitions from the surface of an insulator to an ion are shown in Fig. 3. Electrons involved in the transitions can originate in the conduction band ("hot" electrons excited by the incident beam), surface states, or the valence band. The transition probability depends on the density of occupied electronic surface states, height of the surface barrier, ionic species and charge state, surface-ion separation $(z)$, and ion

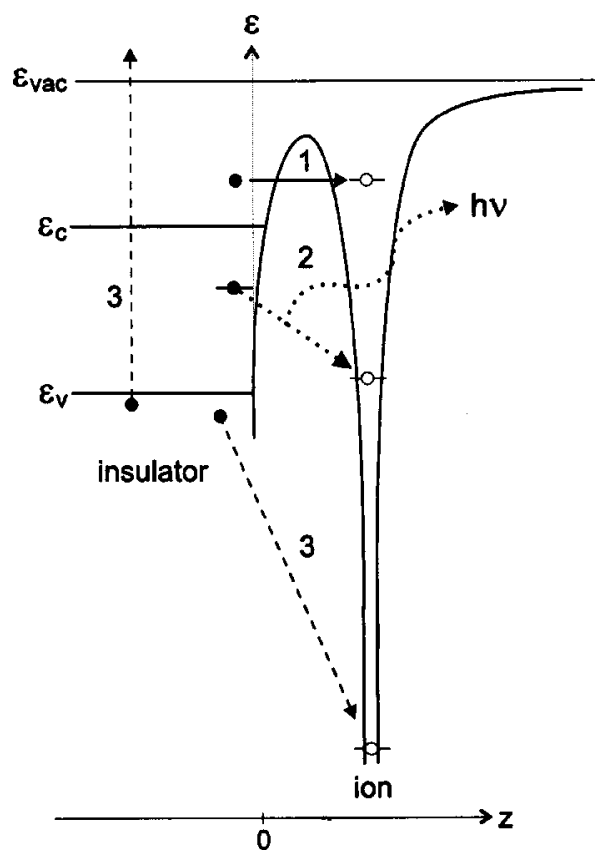

FIG. 3. Schematic illustration of a number of possible transitions between an electron at the surface of an insulator and a gas ion outside the solid surface, 1: resonant capture of a hot electron in the conduction band, 2: radiative capture of an electron in a surface state, and 3: Auger neutralization involving electrons in the valence band. Adapted from Refs. 26-28 ( $\varepsilon_{\mathrm{vac}}$ : vacuum level, $\varepsilon_{c}$ : conduction band minimum, $\varepsilon_{v}$ : valence band maximum, and full and empty circles denote occupied and vacant states, respectively). 
energy. ${ }^{26-28}$ In low vacuum SEMs, such transitions assist in the neutralization of ionized gas molecules and electron irradiated insulators. Auger transitions can also occur, in which an electron in the sample or in the gas molecule is promoted above the vacuum level $\left(\varepsilon_{\text {vac }}\right)$, see transition 3 in Fig. 3. The electron can escape the solid-ion system and then be amplified in the gas cascade, thus contributing to the second Townsend coefficient, $\gamma$ (i.e., the efficiency with which gas ions effectively eject electrons from the sample and introduce a feedback component into cascade amplification). ${ }^{14,23}$ In the case of water vapor, the imaging gas used in this work, the $\gamma$ component of cascade amplification is believed to be negligible. ${ }^{23}$

Gas ions can also recombine with free electrons in the specimen chamber. Recombination with primary and backscattered electrons is negligible due to the energy dependence of the electron capture process ${ }^{30}$ and the high kinetic energies possessed by these electrons. ${ }^{21}$ However, it has been suggested that recombination with SEs may be significant, particularly in the vicinity of the sample surface, before the electrons are appreciably accelerated by the electric field between the sample and the ring electrode. ${ }^{31}$ The rate of recombination between ions and emitted SEs (and hot electrons in the sample which, in the absence of ions, would enter the gas cascade) affects the number of electrons admitted to the cascade. The recombination rate depends on the local ion concentration and on the energy distribution of emitted SEs, both of which can vary across the imaged region of a sample. Consequent effects on the ring electrode imaging signal, $I_{\text {ring }}$ (see Fig. 1), have been ascribed to be the cause of contrast in electron images ${ }^{16,31}$ (these contrast mechanisms are discussed in detail in Sec. IV).

The steady state ion concentration is determined by the ion generation and neutralization rates. The latter is governed by the rates of the above electron-ion recombination processes (and the time constants associated with the drift of ions generated in the sample-electrode gap to the specimen surface). In the case of insulators, time constants associated with ion neutralization rates and the steady state ion concentrations are greater than in the case of grounded conductors. ${ }^{31}$ Electric fields generated by the ions and the relatively large time constants associated with ion neutralization rates have been reported to cause anomalies in SE images of insulators. ${ }^{32,33}$

Finally, large angle PE-gas molecule collisions cause the formation of an electron "skirt" around the unscattered component of the electron beam (see Fig. 1). ${ }^{13,34}$ The skirt is sufficiently delocalized for SEs and BSEs excited by skirt electrons not to affect image contrast generated by the unscattered beam. However, elastic PE-gas scattering reduces the unscattered primary beam current and introduces a non information-carrying, constant background component into SE and BSE imaging signals. ${ }^{13,14,34}$

\section{B. SE emission from uncharged dielectrics}

To a first approximation, the energy loss rate of an electron traversing a dielectric is proportional to the electronhole pair generation rate ${ }^{35,36}$ (plasmons decay into electron-
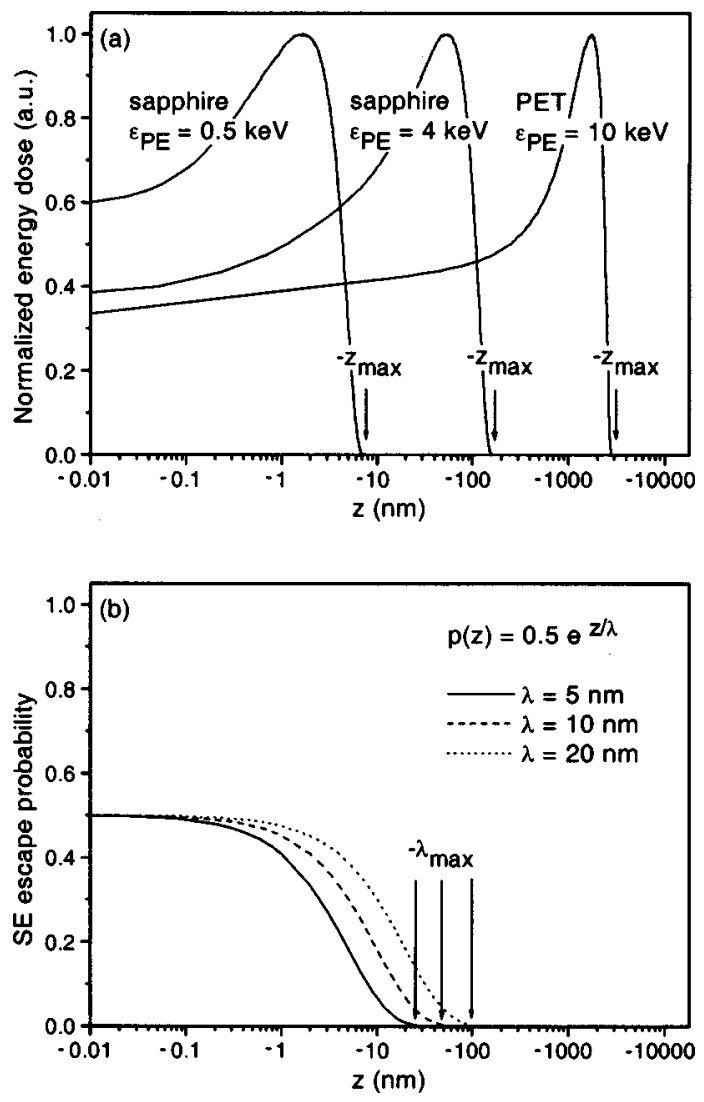

FIG. 4. Hot electron (i.e., SE) generation rate and escape probability profiles: (a) depth resolved primary and backscattered electron energy loss curves calculated for sapphire (density $=3.9 \mathrm{~g} / \mathrm{cm}^{3}$ ) using $\varepsilon_{\mathrm{PE}}=0.5$ and 4 $\mathrm{keV}$, and for PET (density $=0.92 \mathrm{~g} / \mathrm{cm}^{3}$ ) using $\varepsilon_{\mathrm{PE}}=10 \mathrm{keV}$; (b) SE escape probability profiles, $p(z)$, calculated using Eq. (1) $\left(\varepsilon_{\mathrm{PE}}\right.$ : primary beam energy, $z=0$ at the sample surface, $-z_{\max }$ : maximum primary electron penetration range, $\lambda$ : mean SE escape depth, and $\lambda_{\max }$ : maximum SE escape depth).

hole pairs and the rate of energy loss caused by excitation of $x$ rays and phonons is relatively insignificant). In a SEM, the spatial distribution of the generation rate of hot electrons (i.e., SEs) can therefore be approximated by PE and BSE energy loss profiles. ${ }^{10,22,37}$ Figure 4 (a) shows the depth dependence of hot electron generation rates thus calculated for sapphire irradiated with 0.5 and $4 \mathrm{keV}$ electrons, and for polyethylene teraphthalate (PET) irradiated with a $10 \mathrm{keV}$ electron beam (the materials and energies correspond to conditions used to obtain experimental data discussed in Sec. IV). The calculations were performed using the Monte Carlo program CASINO $^{36,38}$ using tabulated elastic Mott cross sections $^{39}$ and the modified expression for the Bethe stopping power. ${ }^{40}$ Each curve is an average of $10^{6}$ primary electron trajectories.

SE emission requires that hot electrons diffuse to the surface and overcome the surface barrier. The probability of SE emission, $p(z)$, therefore decreases with increasing SE generation depth, $-z$. It is usually assumed that ${ }^{10,22}$

$$
p(z)=A e^{z / \lambda},
$$

where $A$ is a constant that accounts for the angular distribution of hot electrons at the sample surface $(A<1), z$ is negative (see Fig. 1), and $\lambda$ is the mean SE escape depth. ${ }^{10,22}$ In 
insulators, $\lambda$ is typically believed to be in the range $10-20$ $\mathrm{nm}$, and the "maximum" SE escape depth $\left(\lambda_{\max }\right)$ is taken to equal $5 \lambda .^{22}$ Figure 4(b) shows $p(z)$ profiles calculated using $A=0.5$, and $\lambda=5,10$, and $20 \mathrm{~nm}$. The SE generation and escape probability profiles shown in Figs. 4(a) and 4(b) illustrate the typical decrease in the rate of SE emission with increasing depth expected for dielectrics imaged in a SEM. ${ }^{10,22,37}$

\section{Charging of dielectrics in high vacuum}

Electron irradiation of an insulator generally leads to a buildup of excess charge due to implantation of incident electrons in the specimen [between the sample surface and $-z_{\max }$, see Fig. 4(a)] and SE emission from the near-surface region shown in Fig. 4(b). ${ }^{10,41-45}$ The polarity of the resulting surface potential essentially depends on the rate at which PEs lose energy as they traverse the sample (the SE generation rate), and on the maximum SE generation depth $\left(z_{\max }\right)$ relative to the maximum SE escape depth $\left(\lambda_{\max }\right){ }^{42-44}$ The maximum SE generation depth is equal to the PE penetration range [see Fig. 4(a)]..$^{10,37,42} \lambda_{\max }$ [see Fig. 4(b)] is governed by the energy distribution of SEs generated by the electron beam, the rate at which the SEs lose energy during diffusion to the surface and by the height of the surface barrier. ${ }^{10,22,37}$ In general (i.e., under conditions of sufficiently high $\varepsilon_{\mathrm{PE}}$ ), the smaller $z_{\max }$, the greater the fraction of hot electrons that can reach the surface with sufficient momentum (component normal to the surface) to leave the sample, and the greater the SE yield $(\delta$, the mean number of SEs emitted per incident electron). If $z_{\max }$ is sufficiently small, the total emissive current can temporarily exceed the current injected into the specimen (since each primary electron is sufficiently energetic to excite a large number of hot electrons), thus giving rise to a positive surface potential. The latter pins some fraction of subsequently generated SEs at the sample surface. A dynamic equilibrium is established when the extent of SE pinning caused by positive sample charging is such that the injected and emissive currents are equal. ${ }^{10,42,44}$ The magnitude of the positive surface potential is self-limited to a few volts since most SEs possess energies of only a few electron volts. $^{10}$

If $z_{\max }$ (i.e., $\varepsilon_{\mathrm{PE}}$ ) is sufficiently large, the injected current can temporarily exceed the total emissive current and give rise to a negative surface potential. ${ }^{10,41-45}$ The net electric field within the SE escape region $\left(-z \leqslant \lambda_{\max }\right)$ accelerates SEs (in the positive $z$ direction, see Figs. 1 and 4), thus causing an effective reduction in the height of the SE escape barrier and an increase in the critical angle for total internal reflection of hot electrons. ${ }^{42,44,45}$ Above the sample surface, the field decelerates the incoming electron beam and lowers the landing energy of primary electrons. ${ }^{10}$ All of these effects contribute to an increase in the SE yield. ${ }^{10,42,44,45}$ A steady state is attained when $\delta(t)$ is such that the total emissive and injected currents are equal. ${ }^{10,44}$

In the case of negative sample charging the surface potential can reach hundreds or thousands of volts and the PE landing energy can be reduced by a corresponding number of electron volts. ${ }^{10}$ Hence in contrast to the case of positive sample charging, the PE penetration range and PE energy loss (i.e., SE generation) profiles are significantly altered by the field produced by trapped electrons, ${ }^{46}$ an effect that was not accounted for in the simulations used to produce the profiles shown in Fig. 4(a). The curves are therefore merely an indication of the SE generation depth profiles at the start of electron irradiation (before the sample charges). Nonetheless, as is discussed in Sec. IV, the curves provide an indication of the $\varepsilon_{\mathrm{PE}^{-}}$-dependence of the polarity of the charge state that a sample converges to after prolonged electron irradiation. Quantitatively, the time evolution and equilibrium magnitude of the charge state are functions of microscope operating parameters, dielectric properties of the specimen, sample-stage-vacuum chamber geometry, and the dynamics of radiation induced conductivity and beam-induced sample modification. ${ }^{43-45,47}$

Above the sample surface, the field generated by subsurface excess charge terminates on conductive objects in the vacuum chamber and therefore modifies the trajectories, as well as the angular and energy distributions of emitted SEs. The field can alter the SE detector collection efficiency ${ }^{42}$ and, in some cases, give rise to image contrast that depends on the sample-detector-vacuum chamber geometry. ${ }^{48}$

\section{EXPERIMENT}

Conventional (high vacuum, $P<10^{-6}$ Torr) SE images were obtained using an Everhart-Thornley detector ${ }^{10,22}$ installed on an FEI Philips XL 30 Field Emission Gun Environmental Scanning Electron Microscope (FEG ESEM). Low vacuum experiments were performed using an environmental secondary detector (ESD) ${ }^{14}$ and a gaseous secondary electron detector (GSED) ${ }^{49}$ installed on an FEI Philips XL 30 FEG ESEM and on an ElectroScan model E3 ESEM. ESD and GSED imaging signals are equivalent to $I_{\text {ring }}$, the signal induced in the ring electrode shown in Fig. 1. The GSED ${ }^{49,50}$ differs from the ESD in that it has been designed to reduce the type III and IV SE components of the imaging signal (i.e., SEs generated by BSE impact on surfaces inside the vacuum chamber, ${ }^{10}$ and SEs generated in ionizing collisions between electrons and gas molecules located above the ring electrode ${ }^{14}$ shown in Fig. 1). Water vapor was used as the imaging gas.

All experiments were performed using PET, polytetrafluoroethylene (TEFLON), sapphire, and muscovite mica specimens. Qualitatively, the presented results are representative of all samples imaged using each of the above detectors.

Images obtained using $I_{\text {ion }}$, current induced in the specimen stage (see Fig. 1) are not discussed in this article. Differences between the $I_{\text {ring }}$ and $I_{\text {ion }}$ imaging signals have been discussed in Refs. 31 and 33.

\section{RESULTS AND DISCUSSION}

We start by illustrating the effects of localized sample charging on contrast in "conventional" SE images obtained using a high vacuum SEM (Sec. IV A). These well understood results are then compared to data obtained from electron irradiated insulators in a low vacuum environment (Secs. IV B and IV C). In Sec. IV D, the presented results are 

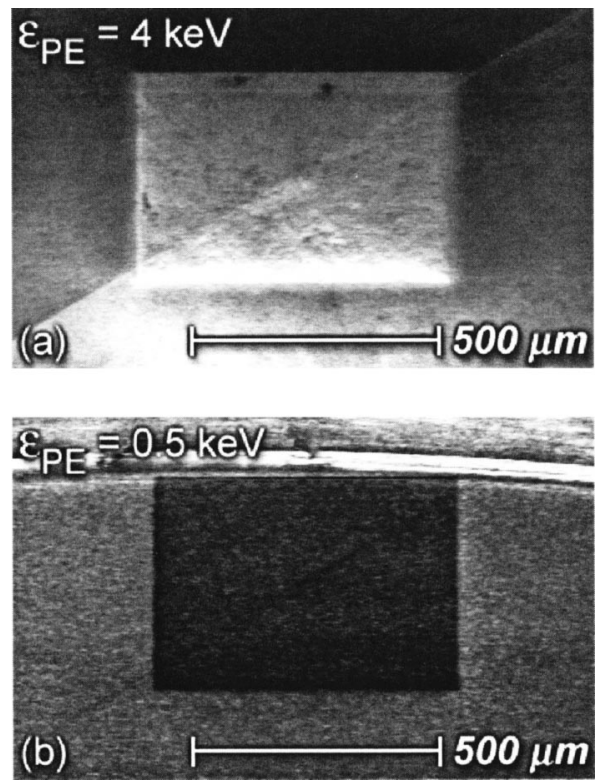

FIG. 5. Secondary electron images of sapphire obtained in high vacuum. The images show contrast produced by localized negative and positive charging. The rectangle in each image was produced by a $5 \mathrm{~s}$ electron beam preirradiation treatment at elevated magnification prior to image acquisition, (a) contrast produced by trapped electrons $\left(\varepsilon_{\mathrm{PE}}=4 \mathrm{keV}\right)$ and (b) contrast produced by trapped holes $\left(\varepsilon_{\mathrm{PE}}=0.5 \mathrm{keV}\right)$. Each image was acquired from a different region of the sample.

used to construct a model of SE emission from dielectrics irradiated by electrons and soft-landing positive ions. The Appendix contains a discussion of aspects of the results that are not critical for the presented model, but need to be commented on for completeness.

It should be pointed out that none of the image contrast effects discussed in this article were observed in BSE images (obtained using an Everhart-Thornley detector operated in passive mode with zero bias on the scintillator). This is ascribed to the high energies possessed by BSEs, ${ }^{21}$ implying that (i) BSE-ion recombination rates, (ii) the electric fields generated by ionized gas molecules, and (iii) the extent of charging exhibited by dielectrics in a low vacuum environment are all too low to perturb the BSE signal significantly, consistent with existing literature ${ }^{13,16,31-33}$ and the model presented in Sec. IV D.

\section{A. Localized charging of dielectrics in high vacuum}

In a high vacuum SEM, insulators can be imaged using low energy primary electron beams $\left(\varepsilon_{\mathrm{PE}} \leqslant \sim 5 \mathrm{keV}\right)$. $^{7,10,42}$ The effects of localized sample charging on SE contrast can be demonstrated using a technique described by Joy and Joy. ${ }^{42}$ First, a localized region containing an elevated concentration of excess trapped charge is produced by irradiating a sample with a scanning electron beam. Second, magnification is reduced and the pre-irradiated region is imaged to show the effects of trapped charge on SE contrast. SE images of sapphire obtained (in high vacuum) using the above procedure are shown in Fig. 5. Images (a) and (b), acquired using beam energies of 4 and $0.5 \mathrm{keV}$, respectively, show how the preirradiated region appears as either a bright or a dark rectangle, illustrating typical SE contrast caused by localized (a) negative and (b) positive charging, respectively. $^{42}$

The PE and BSE energy loss curves calculated for 4 and $0.5 \mathrm{keV}$ electrons shown in Fig. 4(a) approximate the initial hot electron (i.e., SE) generation depth profiles under the conditions used to irradiate the sapphire sample shown in Figs. 5(a) and 5(b), respectively. The SE generation and escape probability profiles shown in Figs. 4(a) and 4(b) show that, when $\varepsilon_{\mathrm{PE}}=0.5 \mathrm{keV}$, all hot electrons are generated within the first $10 \mathrm{~nm}$ of the sample surface, below the expected maximum SE escape depth, $\lambda_{\max }$. As was discussed in Sec. II C, the surface potential can therefore float positive, causing a reduction in $\delta(t),{ }^{10,42,44}$ hence the dark rectangle in the micrograph shown in Fig. 5(b). When $\varepsilon_{\mathrm{PE}}=4 \mathrm{keV}$, the initial hot electron generation depth profile extends beyond $100 \mathrm{~nm}$ and the injected current is temporarily greater than the emissive current. ${ }^{10,42,44}$ The consequent increase in $\delta(t)$ is seen as the bright rectangle in the image shown in Fig. $5(\mathrm{a}) .^{42}$

The SE contrast shown in Fig. 5 is usually dynamic. That is, the contrast due to charge buildup is generally only observed in the first few image frames after the magnification is reduced. During image acquisition, the rastering action of the electron beam causes charge buildup in the (large) imaged region and corresponding changes in $\delta(t)$. It also affects the density of excess charge in the (smaller) preirradiated region. The reason that the contrast is observed at all is that excess charge carriers are trapped at defect sites and the charge induced changes in $\delta(t)$ (i.e., the rates at which a dielectric charges up and discharges) are functions of current density and hence magnification (under appropriate conditions of beam energy, current, and scan speed). ${ }^{17,42,44,45}$

\section{B. Localized charging of dielectrics in low vacuum}

Figure 6(a) shows a GSED image of PET obtained by the procedure used to acquire the micrographs shown in Fig. 5 (i.e., the sample was preirradiated for $5 \mathrm{~s}$ at elevated magnification) using a $10 \mathrm{keV}$ electron beam, a water vapor pressure of 0.4 Torr, and an electrode bias $\left(V_{e}\right)$ of $332 \mathrm{~V}$. The preirradiated region appears as a bright rectangle in the center of the image (the dark left-hand edge and smearing in the image are discussed in the Appendix). The PE and BSE energy loss profile shown in Fig. 4(a) clearly shows that most electrons excited in PET by a $10 \mathrm{keV}$ electron beam are generated well below the maximum SE escape depth [see Fig. 4(b)], indicating that the sample should exhibit negative charging and the preirradiated region should appear bright in SE images, as it does in Fig. 6(a). However, the image contrast was observed to invert if the detector field strength was increased by increasing $V_{e}$ [see Fig. 6(b)], or by decreasing $d$ (defined in Fig. 1); or if the mean free path of ions in the imaging gas was increased by decreasing $P$. That is, under conditions of high field strength and long ionic mean free path, regions that contain elevated concentrations of trapped electrons appear dark in SE images obtained using the signal induced in a biased electrode, despite the increase in the SE yield caused by negative charging. 

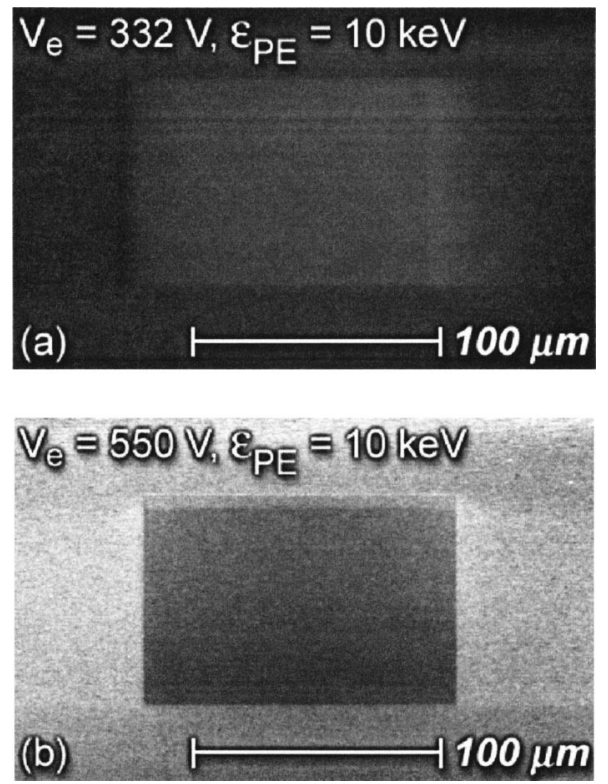

FIG. 6. GSED images of the same region of PET showing contrast produced by localized negative charging. The rectangle in each image was produced by a $5 \mathrm{~s}$ electron beam preirradiation treatment at elevated magnification prior to image acquisition $\left(\varepsilon_{\mathrm{PE}}=10 \mathrm{keV}\right.$, beam dwell time $=2.4 \mu \mathrm{s} /$ pixel, $P=0.4$ Torr, and $d=1.3 \mathrm{~mm})$, (a) normal contrast $\left(V_{e}=332 \mathrm{~V}\right)$ and (b) inverted contrast $\left(V_{e}=550 \mathrm{~V}\right)$.

The above $V_{e}, d$, and $P$ dependence of SE contrast reversal has recently been observed in SE images of topographic asperities on the surface of a grounded conductor (i.e., in the absence of sample charging). ${ }^{31}$ Topographic asperities exhibit elevated SE yields and appear bright in "normal" SE images. ${ }^{10}$ The inversion of topographic contrast under conditions of high detector field strength and long ionic mean free path has been attributed to spatial inhomogeneities in the SE-ion recombination rate at the sample surface. ${ }^{31}$ The cause of such inhomogeneities is illustrated by the diagram in Fig. 7(a) which shows the distribution of electric equipotentials in the vicinity of an asperity on the surface of a grounded conductor (located $1 \mathrm{~mm}$ below a biased electrode, $V_{e}=500 \mathrm{~V}$ ). Also shown in the figure are the corresponding electric field lines indicating the direction of the electrostatic force experienced by ionized gas molecules above the sample surface. Under conditions of high field strength and long ionic mean free path (i.e., when the ion trajectories are not significantly randomized by collisions with gas molecules), the instantaneous directions of the ion velocity vectors are approximately parallel to the local electric field direction, and the ions preferentially drift to regions where the field strength is a maximum. Laterally, the ion concentration is therefore elevated in regions of high field strength (i.e., at topographic asperities). Consequently, these regions exhibit elevated SE-ion recombination rates and can therefore appear dark in SE images (provided the field strength and ionic mean free path are sufficiently large), despite the fact that they exhibit elevated SE yields. ${ }^{31}$

The above argument can also be applied to a flat dielectric that contains a localized region of excess charge. Electric equipotentials calculated for a simplified two-dimensional geometry representing a dielectric (relative permittivity
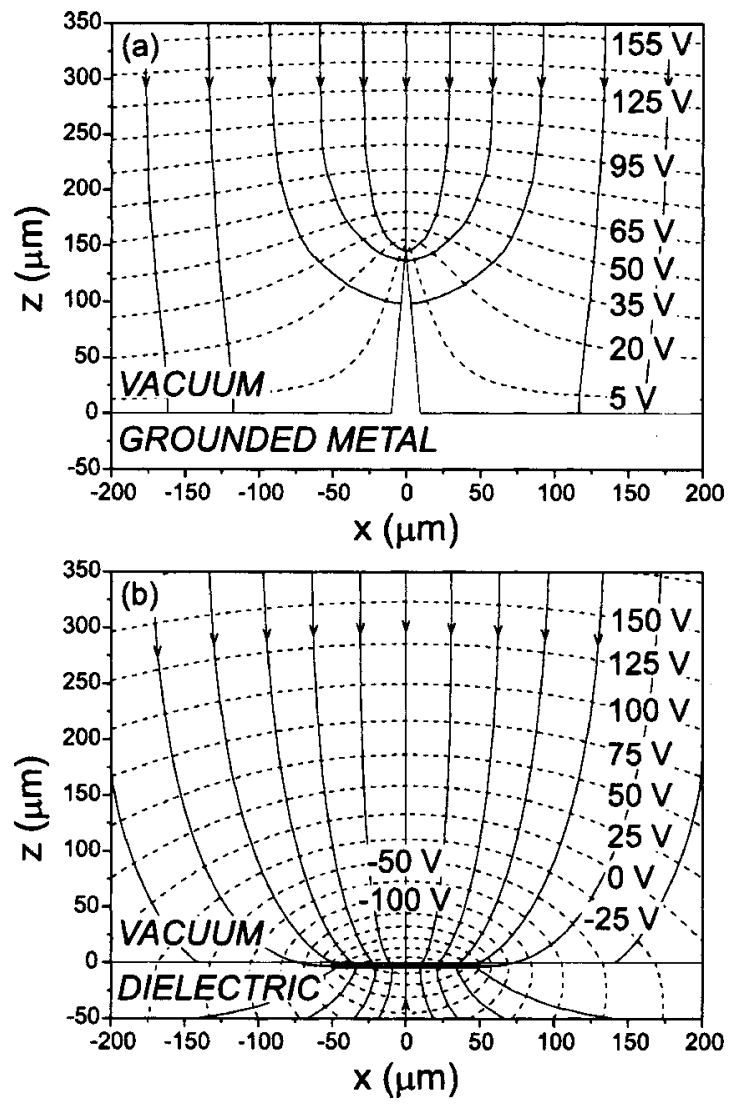

FIG. 7. Electric equipotentials (broken lines) calculated using the finite element software QuickField (Ref. 51) for simplified two-dimensional geometries representing samples under a biased electrode (electrode bias, $V_{e}=500 \mathrm{~V}$; sample-electrode separation, $d=1 \mathrm{~mm}$ ): (a) grounded metal with a topographic asperity and (b) a flat insulator (relative permittivity $=2.1$ ) containing a $4 \times 100 \mu \mathrm{m}$ region of trapped charge (shaded region, charge density $=-22 \mathrm{C} / \mathrm{m}^{3}$ ). Also shown are the electric field lines (full arrows) indicating the direction of the electrostatic force experienced by positive charge carriers.

$=2.1$ ) containing a $4 \mu \mathrm{m}$ deep, $100 \mu \mathrm{m}$ wide region of trapped charge (charge density $=-22 \mathrm{C} / \mathrm{m}^{3}$ ), located $1 \mathrm{~mm}$ below a biased electrode $\left(V_{e}=500 \mathrm{~V}\right)$ are shown in Fig. 7(b). The diagram illustrates that, in the case of an insulator with a localized region that exhibits net negative charging, the intensity of the electric field above the sample surface is a maximum in the vicinity of this region, as in the case of a topographic asperity on the surface of a grounded conductor [see Fig. 7(a)]. When a scanning electron beam impinges on an asperity or on a region that exhibits an elevated concentration of trapped electrons, the SE yield increases and (i) under conditions of low field strength and/or short ionic mean free path, the GSED signal intensity increases and the feature appears bright in an electron image ("normal" contrast), or (ii) under conditions of high field strength and long ionic mean free path, the local SE-ion recombination rate increases, the number of SEs admitted to the gas cascade decreases, and the region can appear dark in GSED images ("inverse" contrast). Such darkening of features in SE images requires that the ion flux be much greater than the flux of SEs that give rise to the normal component of image contrast (i.e., the ion concentration must be sufficiently high so as to suppress the fraction of SEs that give rise to normal 
SE contrast completely). Normal contrast is only produced by electrons excited by the focused fraction of the electron beam (by PEs which are not scattered out of the beam by gas molecules, as was mentioned in Sec. II A). Conversely, the inverse component of image contrast (i.e., the rate of SE suppression via SE-ion recombination) can be contributed to by all ions generated in the gas (ions produced as a result of cascade amplification of PEs; and amplification of SEs and BSEs generated by both the focused component of the electron beam and by the skirt). Under typical low vacuum SEM operating conditions, the total ion flux is generally much greater than the flux of SEs that give rise to normal contrast ${ }^{23}$ (exceptions to this case, encountered under conditions of very low pressure, are discussed in Sec. IV C). The proposed mechanism of contrast inversion is therefore plausible provided the cross section for SE capture by an ion at the sample surface is sufficiently high.

The key point in the above discussion is that, in a low vacuum environment, while localized negative charging serves to enhance SE emission [see Fig. 6(a)], the charged feature can appear dark in an electron image due to preferential recombination of emitted SEs with ions located above the sample surface [see Fig. 6(b)]. Such inverse contrast can not occur in images obtained in a conventional, high vacuum SEM. Features that appear dark in high vacuum SE images as a result of localized charging, such as the rectangle in the image shown in Fig. 5(b), are dark because of the effects of positive sample charging on SE emission, a physically distinct mechanism from the SE-ion recombination processes proposed to be the cause of the dark rectangle in the low vacuum image shown in Fig. 6(b).

We conclude this section by noting that positive charging of samples in a low vacuum environment is not discussed in this article. Investigations of positive localized charging caused by preirradiation of bulk dielectrics by a low energy electron beam were inconclusive due to the large increase in the elastic PE-gas scattering cross section with decreasing beam energy. Consequently, at the low beam energies required for positive sample charging, it was not possible to measure SE contrast over a sufficiently wide range of parameters that affect the ion generation rate and steady state ion concentration. The effects of positive localized charging on SE images can be investigated by high energy electron beam irradiation of semiconductor-dielectric-metal composites which contain appropriately biased components. Such data will be presented elsewhere.

\section{Ion flux dependence of charge-induced contrast}

The low vacuum SE images discussed in Sec. IV B (Fig. 6) were obtained under conditions selected so as to illustrate unambiguously the two types of SE contrast (normal and inverted) caused by localized negative sample charging. In this section we discuss the behavior of charge-induced contrast under conditions of "very low" pressure (i.e., ion flux), whereby the SE signal component that gives rise to inverse contrast is negligible, and "very high" pressure whereby charge-induced contrast is not observed in SE images.
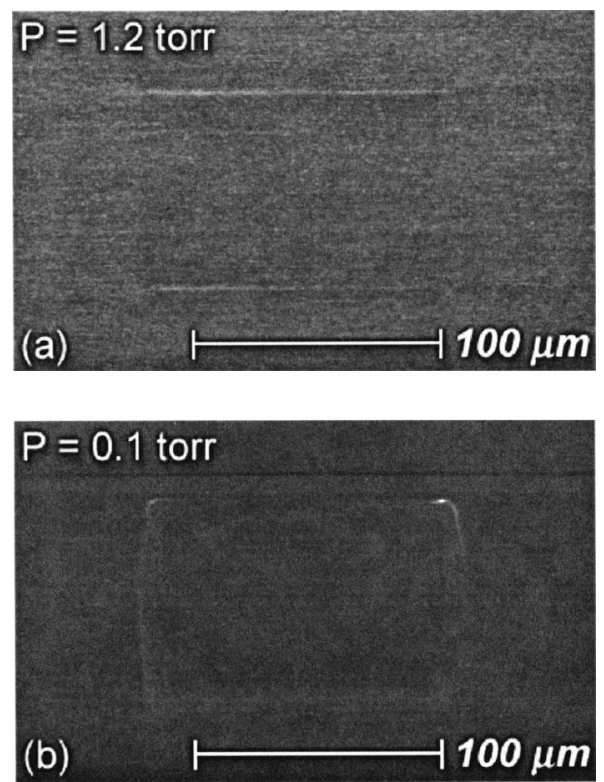

FIG. 8. GSED images of PET showing normal SE contrast produced by negative charging of a dielectric in high and low pressure environments. The rectangle in each image was produced by a $5 \mathrm{~s}$ electron beam preirradiation treatment at elevated magnification prior to image acquisition $\left(\varepsilon_{\mathrm{PE}}=10 \mathrm{keV}\right.$, beam dwell time $=1.3 \mu \mathrm{s} /$ pixel, $V_{e}=550 \mathrm{~V}$, and $d=1.3 \mathrm{~mm})$. Each image was acquired from a different region of the sample.

Figure 8 shows GSED images of regions of PET preirradiated for $5 \mathrm{~s}$ at water vapor pressures of 1.2 and 0.1 Torr. At pressures in excess of $\sim 1.5$ Torr, the preirradiation treatment did not produce SE contrast (not shown in the figure). When $P$ was decreased to $\sim 1.2$ Torr, faint, normal chargeinduced SE contrast was observed in GSED images [Fig. 8 (a)]. As $P$ was decreased to $\sim 0.2$ Torr, the charge-induced contrast became more pronounced and gradually inverted, as in the images shown in Fig. 6, because of the SE-ion recombination effect discussed in Sec. IV B. However, in the experiment shown in Fig. 6, the inversion was caused by a change in field strength, whereas in this case it was caused by the increase in the mean free path of ions in the gas caused by the decrease in $P$. A detailed discussion and examples of such contrast reversal have been presented in Ref. 31 , and will not be reproduced here. When $P$ was decreased below $\sim 0.2$ Torr, the inverse contrast reverted back to normal, and the preirradiated region appeared bright in GSED images [Fig. 8(b)]. The distortions in the shape of the preirradiated rectangle are discussed in the Appendix.

The absence of charge-induced contrast in images obtained at high gas pressures can, in principle, be caused by preferential recombination of ions with excess SEs emitted as a result of sample charging ${ }^{16}$ or by the absence of charging at high pressures. The latter can be excluded on the basis of the results shown in Fig. 9. Regions preirradiated at high pressures did not give rise to charge-induced SE contrast in images acquired at these pressures [Fig. 9(a)], but the contrast was observed in images of the same regions obtained after reducing pressure [Fig. 9(b)]. That is, at high pressures, electron irradiation can cause negative localized charging, but the change in SE emission caused by charging is only detected at low pressures. ${ }^{52}$ 

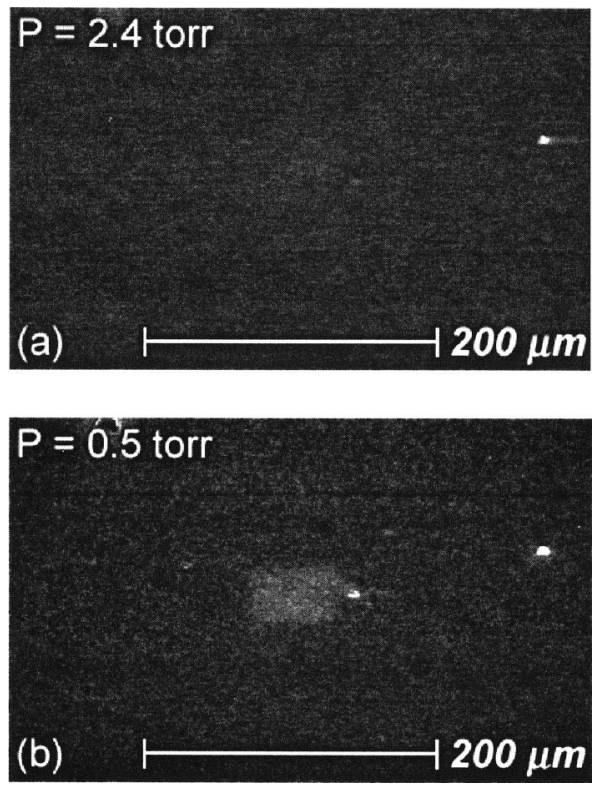

FIG. 9. GSED images of mica showing a region preirradiated for $5 \mathrm{~s}$ at a specimen chamber pressure of 2.4 Torr. Images (a) and (b) were acquired at 2.4 and 0.5 Torr, respectively. The rectangle in the center of image (b) corresponds to the preirradiated region which is not visible in image (a) $\left(\varepsilon_{\mathrm{PE}}=30 \mathrm{keV}\right.$, beam dwell time $=9.4 \mu \mathrm{s} /$ pixel, $d=10.9 \mathrm{~mm}$, and $\left.V_{e}=550 \mathrm{~V}\right)$.

It has been suggested that the absence of charge-induced contrast at high $P$ is a consequence of the energy dependence of the SE-ion recombination rate. ${ }^{16}$ The SE-ion recombination probability rapidly increases with decreasing SE energy. ${ }^{30}$ Variations in the SE energy spectrum can therefore give rise to corresponding variations in the recombination rate. As is argued in Sec. IV D, negative charging causes an increase in the low energy tail of the SE spectrum. These low energy SEs exhibit enhanced SE-ion recombination probabilities and the intensity of the contrast they give rise to is expected to decrease with increasing ion concentration (i.e., pressure), hence the absence of charge-induced contrast at high $P$.

At the opposite extreme, under conditions of very low pressure, negative localized charging gives rise to normal SE contrast in GSED images [see Fig. 8(b)]. This can be explained by the pressure dependence of cascade amplification (i.e., ion generation rate) shown in Fig. 2. The curves illustrate that, in the pressure range of interest, $P<\sim 1$ Torr, the ion generation rate rapidly decreases with decreasing pressure, irrespective of the intensity of the field between the sample and the electrode. Hence, at sufficiently low pressures, the ion concentration and SE-ion recombination rate are too low for suppression of a significant fraction of emitted SEs. Consequently, image contrast is governed by changes in $\delta$ and inverse contrast is not observed in SE images obtained at low pressures [e.g., Fig. 8(b)].

In summary, the pressure (ion flux) dependence of GSED contrast produced by localized negative charging can be classified into four regimes. In order of decreasing pressure: (i) at "very high" pressures charge-induced contrast is not observed in SE images; (ii) at "intermediate" pressures, charging gives rise to normal contrast (as in high vacuum); (iii) at "low" pressures, charging can (under conditions of high field strength) give rise to inverse contrast whereby negative regions appear dark in SE images; and (iv) at "very low" pressures, charging gives rise to normal contrast. The proposed causes of this behavior are directly related to the concentration and lateral distribution of ions in the vicinity of the sample surface.

(i) “Very high" pressure ( $P>1.5-6$ Torr): the generation rate and steady state concentration of ionized gas molecules are very high (see Fig. 2). Accordingly, the SE-ion recombination rate is expected to be high. However, the flux of ions incident on the sample surface is laterally homogeneous because ion trajectories are randomized by frequent collisions with gas molecules. ${ }^{31}$ On the basis of the discussion in Sec. IV B, it therefore follows that the SE-ion recombination rate should be homogeneous and should not affect SE contrast. However, the increase in $\delta$ caused by negative charging is expected to constitute the low energy tail of the SE energy spectrum, as is discussed in Sec. IV D. Hence, due to the Coulombic nature of the SE-ion capture process, ${ }^{30}$ these SEs are much more likely to recombine with ions than SEs emitted due to, for example, surface topography. The intensity of charge-induced SE contrast is therefore expected to decrease with increasing pressure (ion flux) since the ions act as a high-pass SE energy filter.

(ii) "Intermediate" pressure: as $P$ is decreased beyond the regime described in (i), the generation rate and steady state concentration of ions decrease (see Fig. 2). As such, the rates at which SEs recombine with ions decrease. The intensity of normal charge-induced SE contrast therefore increases with decreasing $P$ until the effects described in (iii) start to dominate image contrast.

(iii) "Low" pressure ( $\sim 1>P>\sim 0.2$ Torr): in this pressure regime, provided the intensity of the electric field between the sample and the biased electrode is sufficiently high, ion trajectories are significantly affected by the geometry of the electric field between the sample and the biased electrode. ${ }^{31}$ As such, ions preferentially drift to regions that contain elevated concentrations of excess electrons [see Fig. 7(b)]. These regions therefore exhibit elevated SE-ion recombination rates and can appear dark in GSED images. This contrast mechanism, ascribed to lateral variations in the concentration of ions above the sample surface, should not be confused with the mechanism described in (i), attributed to lateral variations in the energy spectrum of emitted SEs.

(iv) "Very low" pressure ( $P<\sim 0.2$ Torr): the ion concentration is too low to affect image contrast. Normal SE contrast is observed in electron images, as in a high vacuum environment.

Quantitatively, pressures that define the above regimes should only serve as a rough guide. Generic quantification of these regimes is not possible because of the dependence on the steady state ion concentration. The latter is a function of the ion neutralization rate which, even if all other microscope operating parameters are fixed, depends on the electronic properties, size, and shape of the imaged dielectric and on the sample-stage-detector-pole piece geometry. ${ }^{31-33}$ For a given specimen, these regimes can be identified simply by acquiring images as a function of pressure. Inverse contrast 
caused by lateral inhomogeneities in the ion concentration [i.e., pressure regime (iii)] also exhibits the characteristic $V_{e}$ and $d$ (i.e., field strength) dependencies discussed in Section IV B and in Ref. 31.

We should point out that, as regards SE contrast inversion caused by changes in the SEM operating parameters, the above discussion is not exhaustive. For example, there have been reports of contrast reversal ascribed to lateral variations in the charging/discharging rates across the imaged region of a dielectric, ${ }^{17,44}$ the temperature dependence of localized charging, ${ }^{16}$ and to rapid fluctuations in the concentration of ionized gas molecules above the sample surface. ${ }^{33}$

\section{SE emission from dielectrics in low vacuum}

In the existing literature, most examples of chargeinduced SE contrast have been obtained from heterogeneous specimens. The contrast corresponds to lateral variations in trapped charge density. ${ }^{16,17}$ The electron images presented in this article differ from these in the methods used to create inhomogeneous distributions of trapped charge. Here, locally charged regions were produced by filling charge traps during a preirradiation treatment at elevated magnification, whereas in the cited work, such regions result from lateral variations in the charge accumulation and decay rates exhibited by samples irradiated at a given magnification. The contrast observed in the other cases will therefore exhibit different dynamic behavior as a function of PE flux, which is affected by parameters such as scan speed and beam current. However, the present results form a good basis for a generic model of SE emission from a dielectric that exhibits negative charging in a low vacuum environment. We start by considering the vertical $(z)$ component of the electric field inside the insulator, between the surface (at $z=0$ ) and the maximum SE escape depth $\left(-z=\lambda_{\max }\right)$. The field consists of components generated by subsurface trapped charge, positive ions above the sample surface, and the biased electrode. As was shown in Sec. IV B, subsurface charging caused by high energy $(\varepsilon>\sim 4 \mathrm{keV}) \mathrm{PE}$ irradiation gives rise to a negative surface potential, as in the case of high vacuum SEM. That is, the net electric field generated at the surface by the positively charged near-surface region (produced by SE emission and recombination of ions with electrons located in the vicinity of the first monolayer of the surface) and by the underlying trapped electrons ${ }^{29,41,44}$ is dominated by the negative underlayer, effectively giving rise to a SE extraction potential at the surface. The electric fields generated by the steady state distribution of positive ions above the sample surface ${ }^{32}$ and by the positively biased electrode (see Fig. 1) also give rise to a SE extraction potential at the surface. The effect of the net field on a surface-ion system is schematically illustrated in the electron energy diagram shown in Fig. 10. The field causes (i) band bending inside the solid as shown in the figure, (ii) a reduction in the height of the potential barrier between the surface and the ion, $\Delta \varepsilon=\varepsilon_{1^{-}} \varepsilon_{2}$, and (iii) a reduction in the net barrier a SE must surmount to escape the solid-ion system and enter the gas cascade, $\Delta \varepsilon=\varepsilon_{3}-\varepsilon_{4}$. The effect of the field on SE emission is schematically illustrated in Fig. 11. The energy distribution of SEs emitted from an

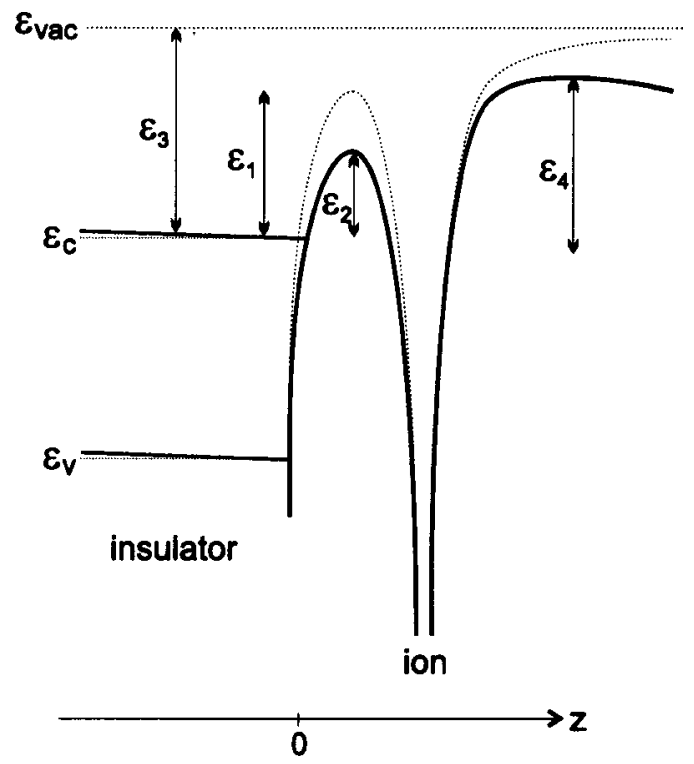

FIG. 10. Simplified electron energy diagram showing an ion incident on the surface of an insulator (broken lines), adapted from Refs. 26-28. Full curves illustrate band bending [within the SE escape region, see Fig. 4(b)] and surface barrier lowering caused by the net electric field generated by (i) negative sample charging, (ii) steady state concentration of positive ions above the sample surface, and (iii) a positively biased electrode above the sample surface ( $z=0$ at the sample surface, $\varepsilon_{\mathrm{vac}}$ : vacuum level, $\boldsymbol{\varepsilon}_{c}$ conduction band minimum, $\varepsilon_{v}$ valence band maximum, $\varepsilon_{1}-\varepsilon_{2}$ : change in the height of the potential barrier between the solid and the ion caused by the field, and $\varepsilon_{3}-\varepsilon_{4}$ : corresponding reduction in the net barrier an electron must surmount to escape the solid-ion system).

uncharged insulator, ${ }^{48,53,54} N_{\mathrm{SE}}(\varepsilon)$, is shown in Fig. 11(a). The corresponding energy spectrum of hot electrons in the solid, $N_{0}(\varepsilon)$, is related to measured $N_{\mathrm{SE}}(\varepsilon)$ spectra through a function of the form: $:^{10,22}$

$$
N_{\mathrm{SE}}(\varepsilon)=N_{0}(\varepsilon) p(\varepsilon) \text {, }
$$

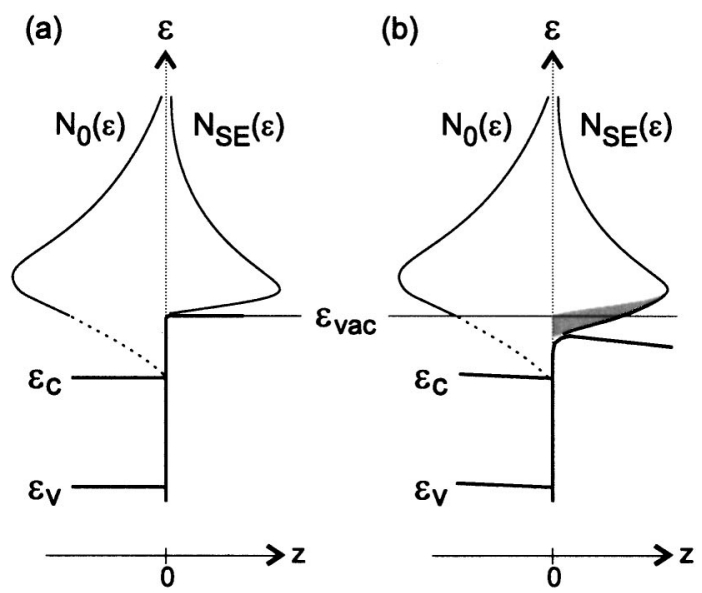

FIG. 11. Schematic illustration of energy distributions of hot electrons excited in an insulator by an electron beam, $N_{0}(\varepsilon)$, and of emitted secondary electrons, $N_{\mathrm{SE}}(\varepsilon)$, in (a) the absence of applied fields and (b) under the influence of the net SE extraction field generated by subsurface trapped charge, gas ions, and a biased electrode. The shaded part of $N_{\mathrm{SE}}(\varepsilon)$ represents the part of the distribution altered by the field $(z=0$ at the sample surface, $\varepsilon_{\mathrm{vac}}$ : vacuum level, $\varepsilon_{c}$ : conduction band minimum, and $\varepsilon_{v}$ : valence band maximum). 
where the SE escape probability, $p(\varepsilon)$, depends on the height and shape of the surface barrier. In the absence of applied fields, $p(\varepsilon)$ is equal to 0 for $\varepsilon \leqslant \varepsilon_{\text {vac }}$ and approaches $B$ as $\varepsilon \rightarrow \infty$ (where the constant $B<1$ due to the angular distribution of hot electrons at $z=0):{ }^{27}$

$$
p(\varepsilon)=B\left[1-\left(\frac{\varepsilon_{\mathrm{vac}}}{\varepsilon}\right)^{\zeta}\right]^{\varsigma} \quad \text { if } \varepsilon \geqslant \varepsilon_{\mathrm{vac}} .
$$

The constants $\zeta$ and $s$ determine the shape of the function between $p(\varepsilon)=0$ and $B$.

The shape of a typical $N_{0}(\varepsilon)$ profile in an uncharged dielectric, deduced from measured SE spectra ${ }^{48,53,54}$ and from Eq. (2), is shown in Fig. 11(a). For energies smaller than $\varepsilon_{\mathrm{vac}}, N_{0}(\varepsilon)$ was extrapolated to the bottom of the conduction band (broken part of the curve), as is discussed below. The total SE yield, $\delta$, is given by ${ }^{10,22}$

$$
\delta=\int N_{\mathrm{SE}}(\varepsilon) d \varepsilon,
$$

where the integration is performed over all possible SE energies. The reduction in the height of the escape barrier due to the applied field (see Figs. 10 and 11) therefore causes an increase in the low energy tail of $N_{\mathrm{SE}}(\varepsilon)$ and an increase in $\delta,^{56}$ as is illustrated by the shaded region of $N_{\mathrm{SE}}(\varepsilon)$ shown in Fig. 11(b).

The field-induced decrease in the height of the surface barrier implies an increase in the maximum SE escape depth, since the escape barrier governs the maximum depth from which SEs generated by the beam can reach the surface with sufficient momentum for emission. This is in direct contradiction to the notion that the information depth of SE contrast due to sample charging in a low vacuum environment is restricted to a few nanometers ${ }^{19}$ (as opposed to the maximum SE escape depth which can exceed tens of nanometers). This theory has been based on the observation that SE images of charged insulators often exhibit unusually high levels of surface contrast (i.e., contrast that corresponds to features located at depths smaller than $\lambda_{\max }$ ), and that charge-induced contrast is not observed in images of samples coated with a thin grounded conductor. ${ }^{19}$ The resolution of the apparent contradiction with the current model is inherent in the $N_{\mathrm{SE}}(\varepsilon)$ profile shown in Fig. 11(b). Surface contrast is largely caused by spatial variations in the height of the surface barrier which governs the energy dependence of the hot electron escape probability, $p(\varepsilon)$. However, the sensitivity of $p(\varepsilon)$ to such variations increases with decreasing electron energy, $\varepsilon$. That is, the emission probability of the low energy SEs that constitute the increase in $\delta$ caused by charging exhibit the greatest sensitivity to subtle variations in the height of the surface barrier, hence the increased amount of surface contrast reported in Ref. 19, despite the expected increase in the maximum SE escape depth. Application of a grounded conductive coating alters SE emission because (i) the electric fields (generated by subsurface trapped charge, ions and the biased electrode) terminate on the film, ${ }^{41}$ (ii) the steady state ion concentration at the surface is reduced since grounded conductors exhibit higher recombination rates between ions and electrons (in the solid, see Fig. 3) than dielectrics, ${ }^{31-33}$ (iii) the maximum $\mathrm{SE}$ escape depth is reduced from tens to a few nanometers since conductors exhibit shorter low energy SE inelastic mean free paths than insulators, ${ }^{24,45,55}$ and (iv) the effects of features that locally affect the SE escape barrier can be significantly modified by the coating. In the context of the present model, it is therefore not surprising that the application of a grounded metallic coating may eliminate surface contrast in images of charged dielectrics.

We note that the increase in emission of low energy SEs indicated in Fig. 11(b) may be underestimated because, as was mentioned above, at energies smaller than $\varepsilon_{\text {vac }}, N_{0}(\varepsilon)$ was extrapolated to the bottom of the conduction band and probably does not represent the true hot electron energy spectrum. Electrons excited below $z=0$ (see Figs. 4 and 11) lose energy as they diffuse to the surface due to inelastic scattering. ${ }^{10}$ However, in insulators, the inelastic mean free path of low energy electrons $(\varepsilon<\sim 10 \mathrm{eV}$ above the conduction band minimum, $\varepsilon_{c}$ ) rapidly increases with decreasing energy. ${ }^{45,55}$ It is therefore reasonable to expect a pile-up of low energy electrons at $z=0$ and a corresponding increase in the low energy tail of $N_{\mathrm{SE}}(\varepsilon)$ under the influence of the extraction field. Furthermore, the extraction field will tend to shift the entire hot electron spectrum to higher energies. However, this effect is not expected to be significant since the maximum SE escape depth, the greatest vertical distance through which a hot electron can be accelerated by the field prior to emission, is only of the order of tens of nanometers (i.e., $\left.\lambda_{\max }\right)$. The large shifts observed in SE spectra of charging insulators in high vacuum $\mathrm{SEMs}^{48,53}$ are caused by acceleration of emitted SEs as they travel through vacuum towards the entrance slit of the SE spectrometer (where the field terminates) located a few milli- or centimeters above the sample surface.

We will now consider the effects of ions on emitted SEs. The interpretation of SE contrast inversion discussed in Sec. IV B implies that, in the vicinity of the sample surface, ions can recombine with a significant fraction of emitted SEs (and hot electrons, located in the sample, which would be emitted in the absence of electron-ion recombination). The capture probability of energetic electrons by ions rapidly increases with decreasing electron energy. ${ }^{30}$ Hence while the electric field generated by the ions contributes to an increase in the low energy tail of $N_{\mathrm{SE}}(\varepsilon),{ }^{56}$ the SE-ion recombination effect preferentially suppresses the number of low energy SEs that are amplified in the gas cascade (and contribute to the imaging signal measured from the ring electrode). The energy dependence of the SE-ion recombination rate accounts for the absence of charge contrast at high pressures (see Sec. IV C). This argument is consistent with recent measurements which have shown that the range of pressures over which charge-induced contrast can be observed in GSED images can be extended by placing an array of grounded metal wires below the ring electrode, just above the sample surface. ${ }^{57}$ This geometry allows for termination of field lines on the wires which act as efficient sinks of positive charge carriers (since grounded conductors exhibit high ion neutralization rates) and thereby limit the ion concentration and the corresponding SE-ion recombination rate.

The net current reaching the ring electrode due to gas amplification of primary and emitted electrons, expressed as 
a function of beam position $(x, y)$, can therefore be written as

$$
I_{\text {ring }}(x, y)=I_{b}+g_{\mathrm{BSE}} I_{\mathrm{BSE}}(x, y)+g_{\mathrm{SE}} I_{\mathrm{SE}}^{\mathrm{amp}}(x, y),
$$

where $I_{b}$ is the non-information-carrying background (image brightness offset) caused by cascade amplification of BSEs and SEs excited by skirt electrons and by amplification of PEs inelastically scattered by gas molecules, ${ }^{13,14,34} g_{\text {BSE }}$ and $g_{\mathrm{SE}}$ are the BSE and SE gas gain factors, respectively, ${ }^{12,23}$ $I_{\mathrm{BSE}}$ is the emitted BSE current, and $I_{\mathrm{SE}}^{\mathrm{amp}}$ is the current of emitted SEs that do not recombine with ions and are amplified in the gas cascade:

$$
\begin{aligned}
& I_{b}=\left(g_{\mathrm{PE}}+g_{\mathrm{BSE}} S \bar{\eta}+g_{\mathrm{SE}} \varrho S \bar{\delta}\right) \cdot I_{\mathrm{PE}}, \\
& I_{\mathrm{BSE}}(x, y)=\eta(x, y) \cdot(1-S) I_{\mathrm{PE}}, \\
& I_{\mathrm{SE}}^{\mathrm{amp}}(x, y)=\varrho(x, y) \delta(x, y) \cdot(1-S) I_{\mathrm{PE}},
\end{aligned}
$$

where $S$ is the fraction of PEs elastically scattered by gas molecules, forming the defocused electron skirt around the beam, ${ }^{13,34} I_{\mathrm{PE}}$ is the primary beam current, $\bar{\eta}$ and $\bar{\delta}$ are the mean BSE and SE yields of the region irradiated by the skirt, $\eta(x, y)$ and $\delta(x, y)$ are the local BSE and SE yields of the region irradiated by the unscattered component of the rastered beam, and $\varrho$ and $\bar{\varrho}$ are the local and mean probabilities that emitted SEs will be amplified in the gas cascade:

$$
\varrho(x, y)=1-\Omega(x, y) \text {, }
$$

where $\Omega(x, y)$ is the SE-ion recombination probability which is a function of the ion concentration at $(x, y)$, in the vicinity of the sample surface.

Neglecting artifacts caused by ion concentration dynamics, ${ }^{32,33}$ the above equations can, in principle, be used to simulate GSED contrast caused by localized charging provided the following effects are accounted for: (i) changes in $\delta(x, y)$ caused by the electric field at the sample surface as is illustrated in Fig. 11, (ii) the lateral and vertical distribution of ions above the sample surface, (iii) the SE-ion recombination probability as a function of SE energy and ion concentration, (iv) evolution of the SE energy spectrum as a function of $z$ due to the field between the sample and the electrode and due to inelastic SE-gas scattering, and (v) perturbations of this field caused by sample charging and the steady state ion distribution. The clearly complex interdependencies of the above effects and dependencies on parameters such as pressure, electrode bias, and the dielectric properties of the sample render such treatment beyond the scope of this article. Future studies of dielectrics under simultaneous electron-ion irradiation will also have to account for sampleand imaging gas-dependent specimen modification caused by electron irradiation ${ }^{45,58-61}$ and ion adsorption/desorption processes. ${ }^{29}$

\section{CONCLUSION}

We performed experiments designed to elucidate SE contrast mechanisms related to localized charging of dielectrics irradiated by a rastered electron beam in a low vacuum environment. The results were used to construct a model that accounts for changes in the surface potential and SE escape

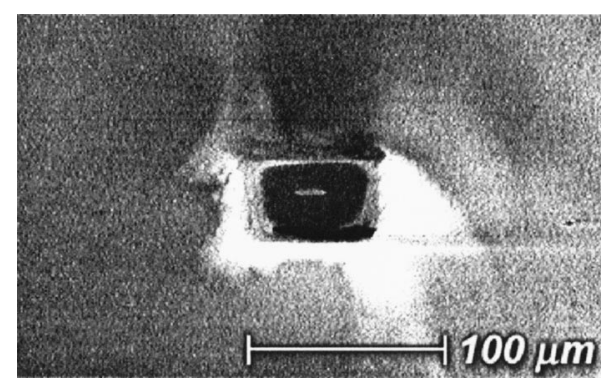

FIG. 12. GSED image of sapphire showing delocalized contrast produced by negative charging $\left(\varepsilon_{\mathrm{PE}}=5 \mathrm{keV}\right.$, beam dwell time $=2.4 \mu \mathrm{s} / \mathrm{pixel}$, $P=0.3$ Torr, $\left.V_{e}=550 \mathrm{~V}, d=2.1 \mathrm{~mm}\right)$.

barrier caused by the electric field generated as a result of simultaneous electron-ion irradiation, and recombination of ions with electrons in the sample and with emitted SEs. The model explains the pressure (i.e., ion flux) dependence of charge-induced SE contrast on the basis of the self-consistent propositions that (i) negative sample charging preferentially enhances the low energy tail of the energy spectrum of emitted SEs, (ii) the energy dependence of SE-ion recombination rates leads to preferential recombination of ions with the low energy SEs that constitute charge-induced contrast, and (iii) the flux and steady state concentration of ions at the sample surface can, under conditions of high field strength and long ionic mean free path, be modulated by the distribution of the electric field above the sample surface, leading to corresponding spatial inhomogeneities in the SE-ion recombination rate which gives rise to an "inverse" SE contrast component. The model predicts an increase in the maximum SE escape depth and accounts for enhanced sensitivity to surface contrast often observed in images of charged dielectrics.

\section{ACKNOWLEDGMENTS}

The authors are grateful for fruitful discussions with Archie Howie, Debbie Stokes, Frank Baker, and John Craven. This work was funded by the EPSRC (Grant No. GR/ M90139) and FEI corporation.

\section{APPENDIX: DYNAMIC CHARGING OF DIELECTRICS IN LOW VACUUM}

The image shown in Fig. 8(b) shows that, at low pressures, the shapes of the rectangular features produced by electron beam preirradiation can be distorted. The features can also be relatively delocalized and dynamic (i.e., the size and shape of the rectangle can change during consecutive acquisition of a number of images). Distorted and delocalized charge-induced contrast is illustrated more clearly by the image of sapphire shown in Fig. 12. The distortions were observed to be most pronounced under conditions of relatively high beam current and low pressure. The distortions can be ascribed to lateral drift of subsurface charge caused by periodic detrapping of trapped carriers due to irradiation by the unscattered component of the rastered electron beam during the preirradiation treatment and during subsequent image acquisition, detrapping resulting from irradiation by the defocused electron skirt (see Fig. 1) which extends be- 


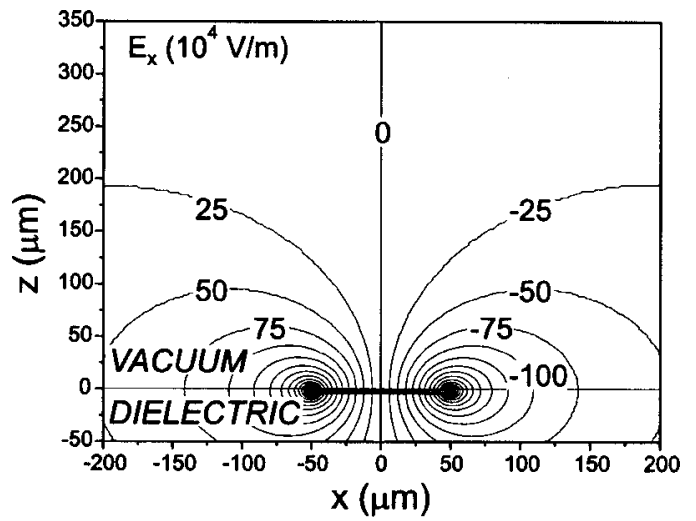

FIG. 13. Spatial distribution of the magnitude of the lateral component of the electric field in and above an insulator containing a $4 \times 100 \mu \mathrm{m}$ region of trapped charge (shaded region, charge density $=-22 \mathrm{C} / \mathrm{m}^{3}$ ), located under a biased electrode (electrode bias, $V_{e}=500 \mathrm{~V}$; sample-electrode separation, $d=1 \mathrm{~mm}$; and relative permittivity $=2.1$, positive $E_{x}$ values indicate an $\mathbf{E}_{x}$ vector pointing in the positive $x$ direction).

yond the imaged region of the sample, drift of de-trapped carriers under the influence of the lateral component of the electric field produced by subsurface charge, and spatial inhomogeneities in the distribution of trapped charge. Carrier detrapping is also expected to be enhanced by subsurface electric fields ${ }^{46}$ and secondary processes resulting from electron irradiation (such as self-absorption of x-rays and cathodoluminescence).

Figure 13 shows the spatial distribution of the magnitude of the lateral component of the electric field $\left(E_{x}\right)$ calculated for an insulator containing a $4 \times 100 \mu \mathrm{m}$ region of trapped electrons, located under a biased electrode. The figure shows that the maximum in the field intensity is at the periphery of the (uniformly) charged region, and the field is directed towards the center of the charged region where $E_{x}=0$. Hence, in between trapping events, detrapped electrons will tend to drift away from the preirradiated region, giving rise to delocalized, dynamic charge-induced contrast in SE images. In real samples, the electric field geometry is more complex due to the positive near surface layer produced by SE emission,${ }^{41-47}$ and distortions in images of preirradiated regions are contributed to by inhomogeneities in charge trap distributions (intrinsic as well as due to beam-induced sample modification), ${ }^{45,58-61}$ and by asymmetries in the raster pattern of the electron beam (the beam dwell time and hence the electron dose exhibit maxima along the left-hand edge and at the top left-hand corner of the imaged region of the sample in order to minimize scan coil instabilities, Ref. 10). Furthermore, any feature present in a SE image of an insulator due to a local increase in the concentration of trapped charge is always embedded in a larger region containing a laterally inhomogeneous density of trapped charge. The latter is caused by irradiation of the sample by the rastered electron beam and by the defocused electron skirt. The skirt intensity decreases with increasing distance from the beam axis,${ }^{13}$ thus further contributing to inhomogeneities in localized charging.

Spatial inhomogeneities in the density of trapped charge will also cause variations in the intensity of the lateral and vertical components of the electric field above the sample surface. As was discussed earlier, the magnitude of the inverse SE contrast component caused by SE-ion recombination scales with the intensity of the electric field between the sample and the biased electrode ${ }^{31}$ [this field is enhanced by subsurface trapped charge as is shown in Fig. 7(b)]. Consequently, the magnitude of the inverse SE contrast component will not be uniform, hence the presence of bright and dark regions within the preirradiated region shown in Fig. 12. A more coherent illustration of this effect can be seen in the image of a preirradiated region of PET shown in Fig. 6(a). The charged region is bright ("normal" SE contrast), except for the left-hand edge which is dark with respect to the surrounding PET. The darkening occurred in the region where the electron beam dwell time is a maximum during the preirradiation treatment (due to the raster sequence of the electron beam). Consequently, this region is expected to contain the greatest density of trapped electrons, a maximum in the local intensity of the electric field produced by trapped charge and a corresponding maximum in the intensity of the inverse SE contrast component caused by SE-ion recombination.

Features in GSED images of insulators often exhibit scan-rate dependent smearing. Such smearing has previously been ascribed to time constants associated with the neutralization rate of ionized gas molecules, and to changes in the ion generation rate during image acquisition (when a scanning electron beam impinges on any feature visible in a SE image, the change in emitted SE current is accompanied by a corresponding change in the ion generation rate due to gas cascade amplification of the emitted SEs). ${ }^{32,33}$ We note that these models have been based on the invalid assumption that the SE-ion recombination rate is negligible. Nonetheless, the models are not inconsistent with the interpretations presented in this article, but will have to be refined to account for the effects of SE-ion recombination on electron imaging signals.

${ }^{1}$ K. T. Chung, J. H. Reisner, and E. R. Campbell, J. Appl. Phys. 54, 6099 (1983).

${ }^{2}$ A. M. Borchert, K. S. Vecchio, and R. D. Stein, Scanning 13, 344 (1991).

${ }^{3}$ S. M. Davidson, in SEM Microcharacterization of Semiconductors, edited by D. B. Holt and D. C. Joy (Academic, London, 1989). p. 153.

${ }^{4}$ M. R. Castell, D. D. Perovic, and H. Lafontaine, Ultramicroscopy 69, 279 (1997).

${ }^{5}$ A. Howie, J. Microsc. 180, 192 (1995)

${ }^{6}$ K. A. Jenkins and B. Oh, J. Appl. Phys. 70, 7179 (1991).

${ }^{7}$ D. M. Taylor, J. Phys. D 11, 2443 (1978)

${ }^{8}$ E. I. Cole, Jr., C. R. Bangell, Jr., B. Davies, A. Neacsu, W. Oxford, S. Roy, and R. H. Propst, J. Appl. Phys. 62, 4909 (1987).

${ }^{9}$ A. Jakubowicz, J. Appl. Phys. 74, 6488 (1993).

${ }^{10}$ L. Reimer, Scanning Electron Microscopy (Springer, Berlin, 1985).

${ }^{11}$ A. B. Harker, J. F. DeNatale, J. F. Flintoff, and J. J. Breen, Appl. Phys. Lett. 62, 3105 (1993).

${ }^{12}$ D. A. Moncrieff, V. N. E. Robinson, and L. B. Harris, J. Phys. D 11, 2315 (1978).

${ }^{13}$ G. D. Danilatos, Adv. Electron. Electron Phys. 71, 109 (1988)

${ }^{14}$ G. D. Danilatos, Adv. Electron. Electron Phys. 78, 1 (1990).

${ }^{15}$ S. Holger and M. W. Füting, Scanning 19, 79 (1997).

${ }^{16}$ M. Toth, S. O. Kucheyev, J. S. Williams, C. Jagadish, M. R. Phillips, and G. Li, Appl. Phys. Lett. 77, 1342 (2000).

${ }^{17}$ D. J. Stokes, B. L. Thiel, and A. M. Donald, Scanning 22, 357 (2000).

${ }^{18}$ T. C. Baroni, B. J. Griffin, J. R. Browne, and F. J. Lincoln, Microsc. Microanal. 6, 49 (2000)

${ }^{19}$ B. J. Griffin, Scanning 22, 234 (2000).

${ }^{20}$ S. Zhu and W. Cao, Phys. Rev. Lett. 79, 2558 (1997).

${ }^{21}$ The primary electron beam energy $\left(\varepsilon_{\mathrm{PE}}\right)$ is typically in the range $1-30$ 
$\mathrm{keV}$. Most backscattered electrons have energies in excess of $0.5 \varepsilon_{\mathrm{PE}}$ (see Ref. 10).

${ }^{22}$ H. Seiler, J. Appl. Phys. 54, R1 (1983).

${ }^{23}$ B. L. Thiel, I. C. Bache, A. L. Fletcher, P. Meredith, and A. M. Donald, J. Microsc. 187, 143 (1997).

${ }^{24}$ A. N. Farley and J. S. Shah, J. Microsc. 158, 389 (1990).

${ }^{25}$ A. Mohan, N. Khanna, J. Hwu, and D. C. Joy, Scanning 20, 436 (1998).

${ }^{26}$ H. D. Hagstrum, Phys. Rev. 119, 940 (1960).

${ }^{27}$ H. D. Hagstrum, Phys. Rev. 122, 83 (1961).

${ }^{28}$ H. D. Hagstrum, Y. Takeishi, and D. D. Pretzer, Phys. Rev. 139, A526 (1965).

${ }^{29}$ D. W. Vance, J. Appl. Phys. 42, 5430 (1971).

${ }^{30}$ Y. Hahn, Rep. Prog. Phys. 60, 691 (1997).

${ }^{31}$ M. Toth, B. L. Thiel, and A. M. Donald, J. Microsc. 205, 86 (2002).

${ }^{32}$ M. Toth and M. R. Phillips, Scanning 22, 313 (2000).

${ }^{33}$ M. Toth and M. R. Phillips, Scanning 22, 370 (2000).

${ }^{34}$ D. A. Moncrieff, P. R. Barker, and V. N. E. Robinson, J. Phys. D 12, 481 (1979).

35 T. E. Everhart and P. H. Hoff, J. Appl. Phys. 42, 5837 (1971).

${ }^{36}$ M. Toth and M. R. Phillips, Scanning 20, 425 (1998).

${ }^{37}$ J. Cazaux, J. Appl. Phys. 89, 8265 (2001).

${ }^{38}$ P. Hovington, D. Drouin, and R. Gauvin, Scanning 19, 1 (1997).

${ }^{39}$ D. Drouin, P. Hovington, and R. Gauvin, Scanning 19, 20 (1997).

${ }^{40}$ D. C. Joy and S. Luo, Scanning 11, 176 (1989).

${ }^{41}$ J. Cazaux, J. Appl. Phys. 59, 1418 (1986).

${ }^{42}$ D. C. Joy and C. S. Joy, Micron 27, 247 (1996).

${ }^{43}$ A. Melchinger and S. Hofmann, J. Appl. Phys. 78, 6224 (1995).

${ }^{44}$ J. Cazaux, J. Appl. Phys. 85, 1137 (1999).

${ }^{45}$ J. Cazaux, J. Electron Spectrosc. Relat. Phenom. 105, 155 (1999).

${ }^{46}$ J. Cazaux, X-ray Spectrometry 25, 265 (1996).
${ }^{47}$ J. Cazaux, Eur. Phys. J.: Appl. Phys. 15, 167 (2001).

${ }^{48}$ M. Belhaj, O. Jbara, S. Odof, K. Msellak, E. I. Rau, and M. V. Andrianov, Scanning 22, 352 (2000).

${ }^{49}$ XL30 ESEM FEG SEM Operating Instructions, FEI Company, Boston, 2000.

${ }^{50}$ A. L. Fletcher, B. L. Thiel, and A. M. Donald, J. Microsc. 196, 26 (1999).

${ }^{51}$ QuickField Finite Element Analysis System, Tera Analysis, Svendborg, 2001.

${ }^{52}$ Images acquired in a low vacuum environment can contain SE contrast caused by subsurface trapped electrons even in the absence of reductions in the PE landing energy (caused by slowing down of PEs above the sample surface by the electric field generated by trapped charge), as has been shown in Ref. 16.

${ }^{53}$ O. Jbara, M. Belhaj, S. Odof, K. Msellak, E. I. Rau, and M. V. Andrianov, Rev. Sci. Instrum. 72, 1788 (2001).

${ }^{54}$ Y. C. Young and J. T. L. Thong, Scanning 22, 161 (2000).

${ }^{55}$ A. Howie, Microsc. Microanal. 6, 291 (2000).

${ }^{56}$ At present, SE spectra of insulators have not been measured in a low vacuum SEM. Such measurements are complicated by the complex geometry of the electric field generated by subsurface trapped charge and gaseous ions, and by the presence of a high concentration of gas molecules in the specimen chamber.

${ }^{57}$ J. P. Craven, F. S. Baker, B. L. Thiel, and A. M. Donald, J. Microsc. 205, 96 (2002).

${ }^{58}$ M. A. Stevens Kalceff and M. R. Phillips, Phys. Rev. B 52, 3122 (1995).

${ }^{59}$ O. Jbara, J. Cazaux, and P. Trebbia, J. Appl. Phys. 78, 868 (1995).

${ }^{60}$ O. Jbara, J. Cazaux, G. Remond, and C. Gilles, J. Appl. Phys. 79, 2309 (1996).

${ }^{61}$ M. Toth, K. Fleischer, and M. R. Phillips, Phys. Rev. B 59, 1575 (1999). 
Journal of Applied Physics is copyrighted by the American Institute of Physics (AIP). Redistribution of journal material is subject to the AIP online journal license and/or AIP copyright. For more information, see http://ojps.aip.org/japo/japcr/jsp

Copyright of Journal of Applied Physics is the property of American Institute of Physics and its content may not be copied or emailed to multiple sites or posted to a listserv without the copyright holder's express written permission. However, users may print, download, or email articles for individual use. 


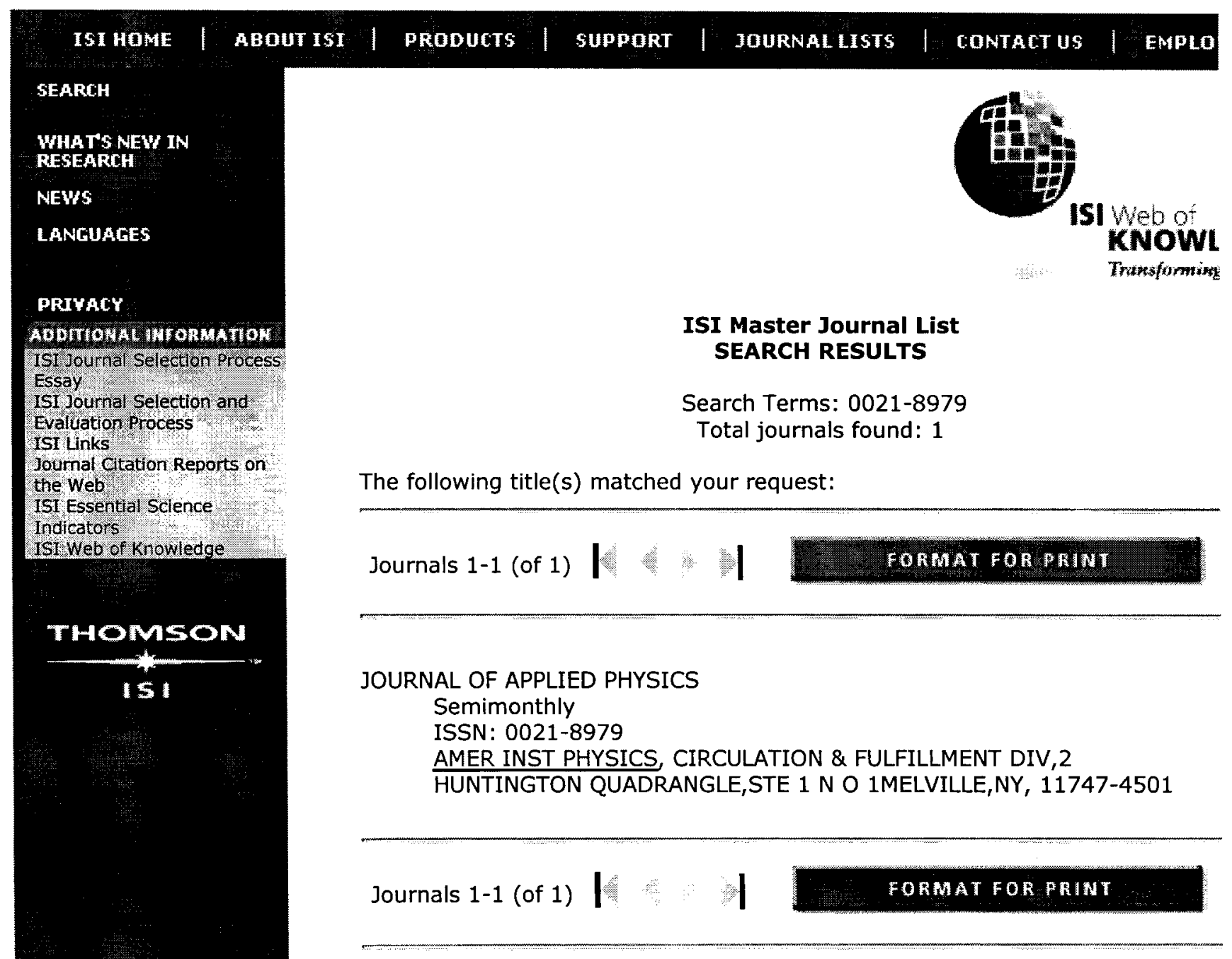

